\title{
Exploring the Pharmacological Mechanisms of Tripterygium wilfordii Hook F against Cardiovascular Disease Using Network Pharmacology and Molecular Docking
}

\author{
Bingwu Huang $\mathbb{D}^{1},{ }^{1}$ Chengbin Huang, ${ }^{2}$ Liuyan Zhu, ${ }^{3}$ Lina Xie, ${ }^{4}$ Yi Wang, ${ }^{5}$ and Ning Zhu $\mathbb{D}^{5}$ \\ ${ }^{1}$ Department of Anesthesiology and Perioperative Medicine, Wenzhou People's Hospital, The Second Affiliated Hospital and Yuying \\ Children's Hospital of Wenzhou Medical University, Wenzhou 325000, Zhejiang Province, China \\ ${ }^{2}$ Department of Orthopedic Surgery, The Second Affiliated Hospital and Yuying Children's Hospital of Wenzhou Medical University, \\ 109 Xueyuan West Road, Wenzhou 325000, Zhejiang Province, China \\ ${ }^{3}$ Department of General Practice, The Wenzhou Third Clinical Institute Affiliated to Wenzhou Medical University, The Third \\ Affiliated Hospital of Shanghai University, Wenzhou People's Hospital, No. 299 Guan Road, Wenzhou 325000, \\ Zhejiang Province, China \\ ${ }^{4}$ Department of Neurosurgery, The Wenzhou Third Clinical Institute Affiliated to Wenzhou Medical University, The Third Affiliated \\ Hospital of Shanghai University, Wenzhou People's Hospital, No. 299 Guan Road, Wenzhou 325000, Zhejiang Province, China \\ ${ }^{5}$ Department of Cardiology, The Wenzhou Third Clinical Institute Affiliated to Wenzhou Medical University, The Third Affiliated \\ Hospital of Shanghai University, Wenzhou People's Hospital, No. 299 Guan Road, Wenzhou 325000, Zhejiang Province, China
}

Correspondence should be addressed to Ning Zhu; zhuningccc@126.com

Received 2 February 2021; Revised 14 June 2021; Accepted 19 July 2021; Published 16 August 2021

Academic Editor: Rita Casadio

Copyright (C) 2021 Bingwu Huang et al. This is an open access article distributed under the Creative Commons Attribution License, which permits unrestricted use, distribution, and reproduction in any medium, provided the original work is properly cited.

\begin{abstract}
Background. Tripterygium wilfordii Hook F (TwHF) has been used in traditional Chinese medicine (TCM) for treating cardiovascular disease (CVD). However, the underlying pharmacological mechanisms of the effects of TwHF on CVD remain elusive. This study revealed the pharmacological mechanisms of TwHF acting on CVD based on a pharmacology approach. Materials and Methods. The active compounds were selected from the Traditional Chinese Medicine Systems Pharmacology (TCMSP) database according to the absorption, distribution, metabolism, and excretion (ADME). The potential targets of TwHF were obtained from the SwissTargetPrediction database. The CVD-related therapeutic targets were collected from the DrugBank, the GeneCards database, and the OMIM database. Protein-protein interaction (PPI) network was generated by the STITCH database. Gene ontology (GO) and Kyoto Encyclopedia of Genes and Genomes (KEGG) pathway enrichment analyses were performed by $\mathrm{R}$ package. The network of drug-targets-diseases-pathways was constructed by the Cytoscape software. Results. The 41 effective ingredients of TwHF and the 178 common targets of TwHF and CVD-related were collected. Furthermore, AKT1, amyloid precursor protein (APP), mitogen-activated protein kinase 1 (MAPK), phosphatidylinositol 3kinase catalytic subunit alpha (PIK3CA), and cellular tumor antigen p53 (TP53) were identified as the core targets involved in the mechanism of TwHF on CVD. Top ten GO (biological processes, cellular components, and molecular functions) and KEGG pathways were screened with a $P$ value $\leq 0.01$. Finally, we constructed the network of TwHF-targets-CVD-GO-KEGG. Conclusions. These findings demonstrate that the main active compound of TwHF, the core targets, and pathways maybe provide new insights into the development of a natural therapy for the prevention and treatment of CVD.
\end{abstract}

\section{Introduction}

Cardiovascular disease (CVD) is a collective term for cardiovascular and cerebrovascular diseases, which is the first cause of death in the world [1]. The burden of CVD is on the rise globally, especially in middle- and low-income countries (LMIC) [2, 3]. In 2013, the World Health Organization (WHO) proposed that countries should reduce premature 


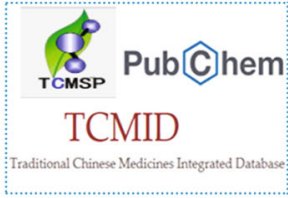

Admet screening

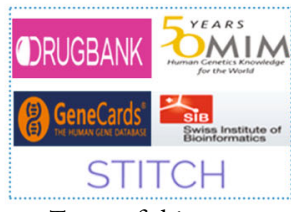

Targets fishing

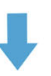

Putative targets

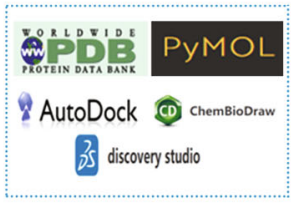

Targets verification

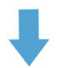

Active compounds

Molecular docking

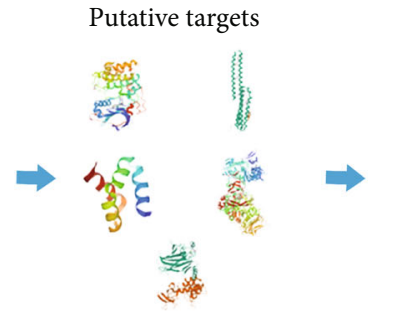

Pharmacological level

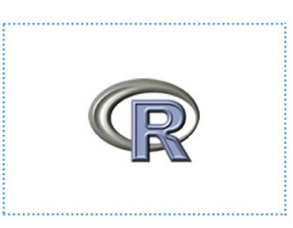

Go and KEGG analysis
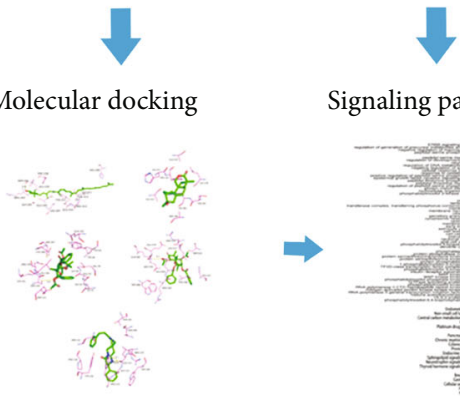

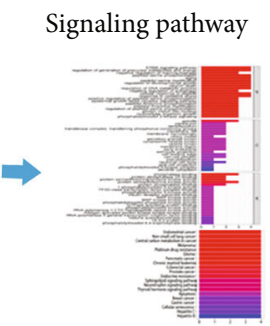

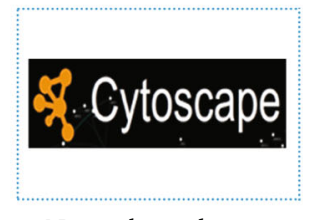

Networks analysis

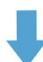

Networks construction

Molecular level

Systems level

Mechanism of action

FIGURE 1: Network pharmacology for deciphering pharmacological mechanisms of Tripterygium wilfordii Hook F acting on cardiovascular disease.

mortality that related to noncommunicable diseases, including CVD, by $25 \%$ by 2025 [4]. Although Western medicines have made good progress in reducing the risk of cardiovascular events and total mortality, patients with long-term cardiovascular treatment still have difficult adherence that might lead to discontinuation of these drugs. This can be attributed to the adverse reactions caused by multiple pharmacologic agents and some drugs that beyond the affordability of LMIC $[3,5]$. Traditional Chinese medicine (TCM), with thousands of years of history in China, has gained widespread clinical applications. In particular, TCM occupies a special position in their heart of the elderly. As a critical component of complementary and alternative medicine, TCM medications have been used for the prevention and treatment of CVD [6].

Tripterygium wilfordii Hook F (TwHF), also known as Leigongteng and Thunder God Vine, has possessed many pharmacological activities such as anticancer, anti-inflammation, antifibrosis, antiatherosclerosis, and antiautoimmune disorders [7-9]. Recently, several fundamental researches have indicated that low-dose TwHF can prevent cardiovascular diseases. Low-dose TwHF can improve the inflammatory reaction, reduce myocardial injury, and optimize acute coronary syndrome (ACS) rat's condition with inhibition of myocardial apoptosis [10]. TwHF extracts were shown to have cardioprotection effects by inducing the activation of Nrf2/HO-1 defense pathway, inhibiting the activation of $\mathrm{NF}-\kappa \mathrm{B}$ pathway and reducing the expression of NLRP3 inflammasome [11-13]. In addition, the extracts can not only improve the vascular function in atherosclerosis, but also may help in the prevention of in-stent restenosis formation following endovascular treatment of lowerextremity artery disease $[14,15]$. However, the underlying pharmacological mechanisms of the effects of TwHF on CVD remain elusive.

Network pharmacology is an innovative way to analyze the complicated relationship between drugs and disease at the system level, which can provide clues for discovering new drugs [16]. This approach integrates and constructs the complicated networks among drug targets, disease targets, and biological processes [17]. It is possible to reveal potential drug-target-disease interactions and realize novel therapeutic application beyond the TCM application through network pharmacology [18]. In this study, target prediction, pharmacokinetic evaluation, molecular structure, biological function, and pathway analysis using many available public databases and bioinformatics tools have systematically elucidated the mechanisms of therapeutic effects of TwHF on CVD (Figure 1).

\section{Materials and Methods}

2.1. Active Component Screening. Traditional Chinese Medicine Systems Pharmacology database (TCMSP, https:// tcmspw.com/tcmsp.php) is an efficient pharmacology resource, which can be used to assess the pharmacokinetics of TCMs or related compounds [19]. It can provide the absorption, distribution, metabolism, and excretion (ADME) properties of compounds, the main indicators of which are oral bioavailability $(\mathrm{OB})$ and drug similarity (DL). OB is a reliable indicator to evaluate the intrinsic quality of drugs objectively, which represents to the speed and degree of absorbing drugs into the circulatory system. And DL represents the sum of the pharmacokinetic properties and safety of compounds, which is calculated by comparing the functional or physical properties of the compounds with those of the majority of known drugs [20]. In this paper, the compound name "Leigongteng" was inputted to the TCMSP database, and active ingredients with $\mathrm{DL} \geq 0.18$ and $\mathrm{OB} \geq$ $30 \%$ were selected for subsequent analysis. Then, SMILES and PubChem ID of candidate components were collected by using the Traditional Chinese Medicines Integrated Database (TCMID, http://www.megabionet.org/tcmid/) [21] and 
the PubChem (https://pubchem.ncbi.nlm.nih.gov/) database [22].

2.2. Identified and Predicted Targets of TwHF. The targets of active components in TwHF were obtained from the SwissTargetPrediction (http://www.swisstargetprediction.ch), which is a free public resource used to accurately predict targets for bioactive molecules [23]. The therapeutic targets of active ingredients were predicted by inputting these components SMILES into SMILES string (s) and searching for their similar molecules. Within the range of "Homo sapiens," high probability targets (probability $P<0.05$ ) were collected after duplicate contents were removed.

\subsection{Target Identification of Known Therapeutic Targets} Acting on CVD. The CVD-related therapeutic targets were collected from the DrugBank (http://www.drugbank.ca) [24], the OMIM database (https://omim.org) [25], and the GeneCards database (https://www.genecards.org) [26]. DrugBank is a freely available network database, which provides molecular information about drugs, drug targets, drug effects, and drug interactions. OMIM database, a comprehensive web resource, is focusing on genes, genetic phenotypes, and their relationships. In addition, GeneCards is a public database that provides detailed information on annotated and predicted genes. With "cardiovascular disease" as the keyword, CVD-related targets were searched among the three databases.

2.4. Protein-Protein Interaction (PPI) Network Construction and Analysis. The identified targets were uploaded to the STITCH database v5.0 (http://stitch.embl.de/) [27] to build the protein-protein interaction network and clarify the functional and physical association between them. The protein interactions were limited to a combined score of 0.9 or higher. The core target genes were determined based on the criterion of combinedscore $\geq 0.9$ and the number of interactions.

2.5. GO and KEGG Pathway Enrichment Analyses. GO analysis can supply gene product biological function information and divide candidate targets into various functional modules, including cellular components (CCs), biological pathways (BPs), and molecular functions (MFs) [28]. KEGG analysis can give functional meaning to genes at molecular or higher levels [29]. Enrichment analyses of GO of core target genes and KEGG were performed by using R (version 3.6.0 for Windows). By using a cut-off value adjusted to $P<0.05$, the top ten GO enrichments and KEGG pathways were screened.

2.6. Construction of Network Relationships. Cytoscape is a free application software, which can transform biomolecular interaction networks into a versatile and interactive visualization framework [30]. The core targets of TwHF on CVD were constructed for KEGG-GO enrichment visualization by the Cytoscape (v3.7.1) software [31]. In the interactive network, the nodes include TwHF, CVD, and their core targets, GO and KEGG pathways. Then, the edges represent the interaction between them.
2.7. Molecular Docking. The crystal structures of target proteins were collected from the RCSB Protein Data Bank (http://www.pdb.org/) and decorated by removing the ligands and water motifs, adding hydrogen, and optimizing the mutation sites by the PyMOL (version 2.3). The 3D chemical structural formulas of key ingredients were collected from PubChem and energy minimized by using ChemBioDraw 3D (version 14). The sites, binding ability, and interactions between compounds and targets were analyzed by PyMOL, AutoDockTools (version 1.5.6), and Discovery Studio 2020 Clients [32, 33]. Autodock vina (1.1.2) was used to conduct docking between compounds and target proteins.

\section{Results}

3.1. Active Ingredient Screening. Total of 51 effective ingredients of TwHF that satisfied $\mathrm{DL} \geq 0.18$ and $\mathrm{OB} \geq 30 \%$ were screened from TCMSP. But the structural formula of 41 effective ingredients of TwHF could be obtained from PubChem (https://www.ncbi.nlm.nih.gov/pccompound) for subsequent analysis (Table 1).

3.2. Target Identification of TwHF and CVD. Firstly, a total of 827 candidate targets of TwHF were downloaded from SwissTargetPrediction (Supplementary Table 1). Secondly, 76 known CVD-related targets were downloaded from the DrugBank database, 358 known CVD-related targets were downloaded from the GeneCards database, and 474 known CVD-related targets were downloaded from the OMIM database (Supplementary Table 1). Then, 802 CVD-related targets were identified by removing the repeated targets. Finally, the 178 common targets of the targets of CVDrelated and TwHF were selected for subsequent analysis (Supplementary Table 1).

3.3. PPI Network Construction and Analysis. Firstly, the PPI network was generated by uploading these 178 identified targets to the STITCH database, and screening condition was limited to combined score $\geq 0.9$. Then, AKT1, amyloid precursor protein (APP), mitogen-activated protein kinase 1 (MAPK), phosphatidylinositol 3-kinase catalytic subunit alpha (PIK3CA), and cellular tumor antigen p53 (TP53) were identified based on the number of interactions (Table 2). These five genes were considered the key putative targets involved in the effects of TwHF on CVD. The raw data (combined score $\geq 0.9$ ) was shown in Supplementary Table 2.

3.4. GO and KEGG Pathway Enrichment Analyses. The 178 candidate targets were selected for GO and KEGG pathway enrichment analyses. The top ten GO analyses of biological process (BP), cellular component (CC), and molecular function (MF) categories were screened (Figure 2). As the results of GO enrichment, the enriched biological process categories were dominated by ERBB signaling pathway, regulation of generation of precursor metabolites and energy, peptidylserine phosphorylation, aging, peptidyl-serine modification, regulation of developmental growth, neuron death, regulation of DNA metabolic process, cellular response to peptide, 
TABLE 1: A list of the final selected compounds from TwHF for network analysis.

\begin{tabular}{|c|c|c|c|}
\hline Molecule ID & Molecule name & OB (\%) & $\mathrm{DL}$ \\
\hline MOL003233 & Triptofordin B2 & 107.71 & 0.76 \\
\hline MOL003209 & Celallocinnine & 83.47 & 0.59 \\
\hline MOL003188 & Tripchlorolide & 78.72 & 0.72 \\
\hline MOL003206 & Canin & 77.41 & 0.33 \\
\hline MOL003225 & Hypodiolide A & 76.13 & 0.49 \\
\hline MOL003279 & $99694-86-7$ & 75.23 & 0.66 \\
\hline MOL003208 & Celafurine & 72.94 & 0.44 \\
\hline MOL003244 & Triptonide & 68.45 & 0.68 \\
\hline MOL005828 & Nobiletin & 61.67 & 0.52 \\
\hline MOL002058 & 40957-99-1 & 57.2 & 0.62 \\
\hline MOL003217 & Isoxanthohumol & 56.81 & 0.39 \\
\hline MOL003224 & Tripdiotolnide & 56.4 & 0.67 \\
\hline MOL000211 & Mairin & 55.38 & 0.78 \\
\hline MOL003187 & Triptolide & 51.29 & 0.68 \\
\hline MOL003280 & Triptonolide & 49.51 & 0.49 \\
\hline MOL003185 & $\begin{array}{l}\text { (1R,4aR,10aS)-5-hydroxy-1-(hydroxymethyl)-7-isopropyl-8-methoxy-1, } \\
\text { 4a-dimethyl-4,9,10,10a-tetrahydro-3H-phenanthren-2-one }\end{array}$ & 48.84 & 0.38 \\
\hline MOL003248 & Triptonoterpene & 48.57 & 0.28 \\
\hline MOL003196 & Tryptophenolide & 48.5 & 0.44 \\
\hline MOL003211 & Celaxanthin & 47.37 & 0.58 \\
\hline MOL003267 & Wilformine & 46.32 & 0.2 \\
\hline MOL003184 & $81827-74-9$ & 45.42 & 0.53 \\
\hline MOL011169 & Peroxyergosterol & 44.39 & 0.82 \\
\hline MOL000449 & Stigmasterol & 43.83 & 0.76 \\
\hline MOL003245 & Triptonoditerpenic acid & 42.56 & 0.39 \\
\hline MOL000422 & Kaempferol & 41.88 & 0.24 \\
\hline MOL003231 & Triptoditerpenic acid B & 40.02 & 0.36 \\
\hline MOL003232 & Triptofordin B1 & 39.55 & 0.84 \\
\hline MOL000296 & Hederagenin & 36.91 & 0.75 \\
\hline MOL000358 & Beta-sitosterol & 36.91 & 0.75 \\
\hline MOL003222 & Salazinic acid & 36.34 & 0.76 \\
\hline MOL003189 & Wilforlide A & 35.66 & 0.72 \\
\hline MOL003229 & Triptinin B & 34.73 & 0.32 \\
\hline MOL003266 & 21-Hydroxy-30-norhopan-22-one & 34.11 & 0.77 \\
\hline MOL003238 & Triptofordin F1 & 33.91 & 0.6 \\
\hline MOL003239 & Triptofordin F2 & 33.62 & 0.67 \\
\hline MOL003278 & Salaspermic acid & 32.19 & 0.63 \\
\hline MOL003235 & Triptofordin D1 & 32 & 0.75 \\
\hline MOL003241 & Triptofordin F4 & 31.37 & 0.67 \\
\hline MOL003242 & Triptofordinine A2 & 30.78 & 0.47 \\
\hline MOL003236 & Triptofordin D2 & 30.38 & 0.69 \\
\hline MOL003210 & Celapanine & 30.18 & 0.82 \\
\hline
\end{tabular}

and response to oxidative stress. CC analysis showed that the spindle was mainly accounted for the largest proportion. The enriched MF categories were dominated by phosphatase binding and protein serine/threonine kinase activity. The KEGG pathway analysis showed that these targets were mainly associated with cancer, melanoma, platinum drug resistance, glioma, chronic myeloid leukemia, endocrine resistance, sphingolipid signaling pathway, neurotrophin signaling pathway, thyroid hormone signaling pathway, apoptosis, cellular senescence, hepatitis C, and hepatitis B (Figure 3).

3.5. Construction of Network. The network visualization of TwHF-targets-CVD-GO-KEGG was generated by using the Cytoscape software (Figure 4). 
TABLE 2: A list of the key putative targets involved in the effects of TwHF on CVD.

\begin{tabular}{lc}
\hline Name & The number of interactions \\
\hline PIK3CA & 50 \\
AKT1 & 36 \\
APP & 34 \\
TP53 & 34 \\
MAPK1 & 31 \\
\hline
\end{tabular}

3.6. Molecular Docking. The crystal structures of potential targets, including AKT1 (PDB: 6CCY; $2.18 \AA$ ), APP (PDB: 5BUO; $2.31 \AA$ ), MAPK1 (PDB: 6SIG; $1.58 \AA$ ), PIK3CA (PDB: 4TTU; $2.18 \AA$ ), and TP53 (PDB: 6RZ3; $4.23 \AA$ ) were collected (Figure 5). Other detail of protein structure could be also found in RCSB Protein Data Bank. Figure 5 showed celaxanthin binds to AKT1 with a binding pocket consisting of SER-240 (2.9 $\AA$ ); hypodiolide A fails to bind to APP without a binding pocket; triptofordin $\mathrm{B} 2$ binds to MAPK1 with a binding pocket consisting of SER-153 (3.3 $\AA$ ) and ARG-155 (3.3 $\AA$ ); triptofordin $\mathrm{B} 2$ binds to PIK3CA with a binding pocket consisting of GLN-582 (3.1 $)$; c celallocinnine binds to TP53 with a binding pocket consisting of LEU-111 (3.2 and $3.0 \AA$ ), ASN-131 (3.1 ̊), and TYR-126 (2.9 ̊).

\section{Discussion}

There is an urgent need to promote new drugs for CVD treatment because of the heavy burden of CVD and the poor efficacy and side effects of the currently used medicines. Network pharmacology was applied to reveal the interaction between medicines and targets of diseases, and it can comprehensively describe the complexity between drugs and diseases $[33,34]$. Therefore, the use of network pharmacology uncovering multiple drug-target interactions may contribute to novel drug discovery in complex diseases such as CVD. TwHF exhibits therapeutic efficacy in preclinical models of CVD and has been identified in several studies [35-37]. In the present study, the underlying mechanism of the protective effects of TwHF on CVD was uncovered by a network pharmacology strategy. Therapeutic targets and signaling pathways were investigated by database screening, PPI network construction, and pathway enrichment analysis. Furthermore, to validate the specific interactions between core targets and CVD, molecular docking was conducted.

In this study, 41 active compounds of TwHF were determined based on ADME. Pharmacological analysis suggested that these active components may have protective effects against CVD. Nobiletin has been reported to attenuate hypoxia/reoxygenation-induced injury by the inhibition of oxidative stress and apoptosis in H9c2 cardiomyocytes, as well as myocardial ischemia and reperfusion injury in vivo $[38,39]$. Triptonide ameliorates diabetic cardiomyopathy via mediating inflammation $[40,41]$. Isoxanthohumol regulates vivo vascular proliferation and in vivo-the inflammatory crosstalk of vascular cells, contributing to the treatment of angiogenesis and inflammation-related diseases [42]. Stigmasterol blocked Ang II-induced aortic smooth muscle cell proliferation by the arrest of the cell-cycle and promoted apoptosis and ROS production [43]. Kaempferol attenuates cardiac hypertrophy and isoproterenol-induced heart failure in diabetic rats $[44,45]$.

Subsequently, the targets of TwHF and CVD were also identified. 178 common candidate targets between TwHF and CVD were selected. Finally, we screened 5 core candidate genes for further analysis. The interactive values and interaction indicate that these targets are closely contacted with other targets in "CVD-target PPI network" and are responsible for TwHF acting on CVD and the pathogenesis of CVD. As was well known, Akt signaling plays an important role in many processes of CVD pathology such as atherosclerosis, vascular remodeling, and cardiac hypertrophy. Several Akt inhibitors have been proven to be potential novel therapeutics for the CVD [46]. PIK3R1, MAPK1, and PIK3CA may modulate platelet activation and be involved in CVD [47]. Class I phosphatidylinositol 3-kinases (PI3Ks) are composed of a regulatory subunit (p85 regulatory subunit) and a catalytic subunit (p110 catalytic subunit) [48, 49]. The catalytic subunit $\mathrm{p} 110 \alpha$ of PI3K is encoded by the gene PIK3CA, which regulates doxorubicin-induced cardiotoxicity [50]. Indeed, the compounded cardiovascular risk of PI3K $\alpha$ inhibitor use in breast cancer is particularly relevant given the prevalence of p110 $\alpha$ gain-of-function mutations [51]. p38 mitogen-activated protein kinase (p38), extracellular signalregulated kinase1/2 (ERK), and c-Jun NH2 terminal protein kinase (JNK) are major components of MAPK kinases, which control embryogenesis, differentiation, proliferation, and death [52]. The inactivation of JNK, p38MAPK, and ERK1/2 could block vascular smooth muscle cell proliferation and migration [53-55]. APP is associated with the adhesion of platelets to amyloid peptides and thrombus formation [56, 57]. It was reported TP53 can differentiate patients with left main coronary artery disease from healthy participants [58].

Top ten GO of each category (BP, MF, and CC) and KEGG pathways associated with TwHF acting on CVD were classified. These categories composed of the most key targets are considered specific and meaningful enrichment. The data showed that the major components were mainly related with multiple BPs, such as ERBB signaling pathway, regulation of generation of precursor metabolites and energy, peptidylserine phosphorylation, aging, peptidyl-serine modification, regulation of developmental growth, neuron death, regulation of DNA metabolic process, cellular response to peptide, and response to oxidative stress. It is reported that ERBB signaling can regulate cardiovascular development and multiple cardiac cell biology [59, 60]. Regulation of developmental growth and regulation of DNA metabolic process suggested TwHF acting on CVD through cardiovascular proliferation. However, BPs including precursor metabolites and energy, peptidyl-serine phosphorylation, neuron death, and cellular response to peptide have not been well investigated. Further studies should be conducted to determine the role of these BPs. The major components acting on CVD were also associated with spindle in terms of CC, which indicated TwHF acting on CVD was associated with regulation of the proliferation and differentiation of cardiovascular cells. The enriched 


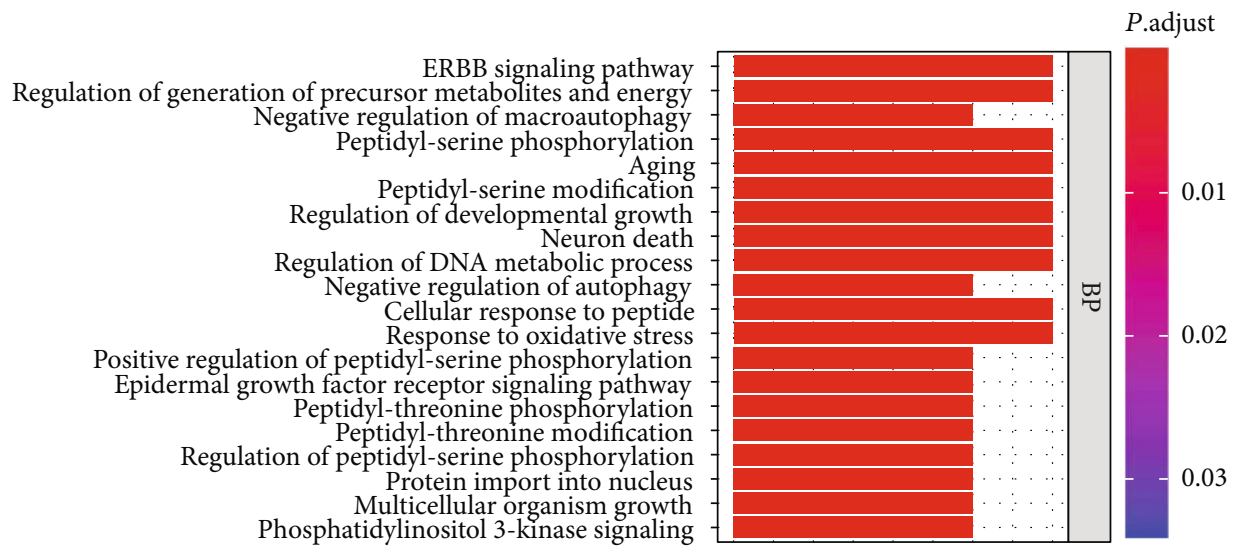

(a)

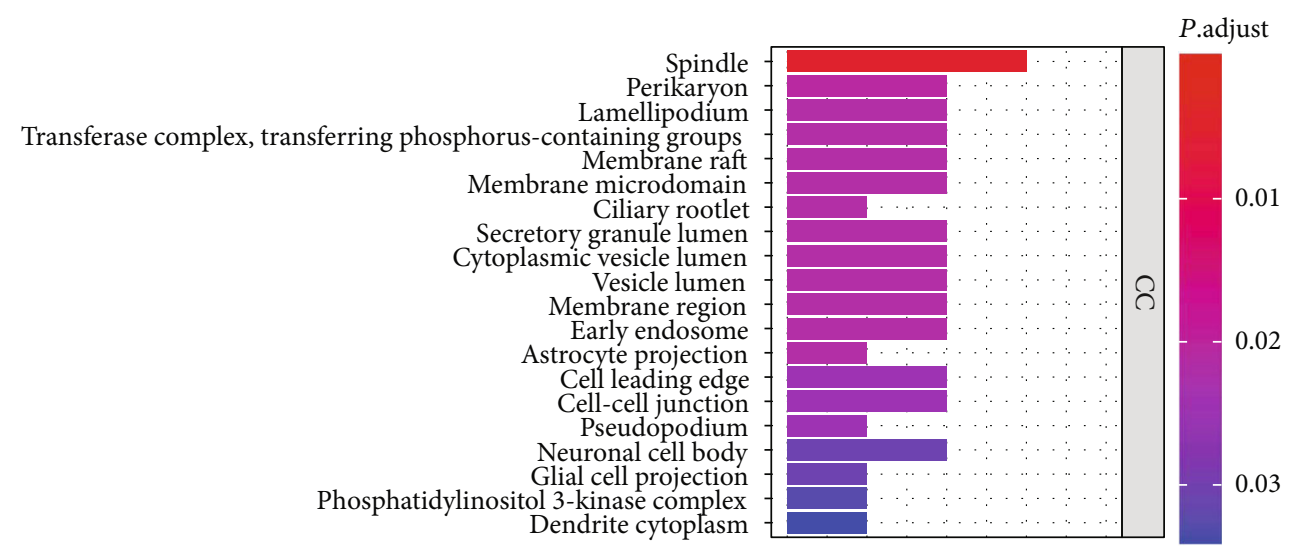

(b)

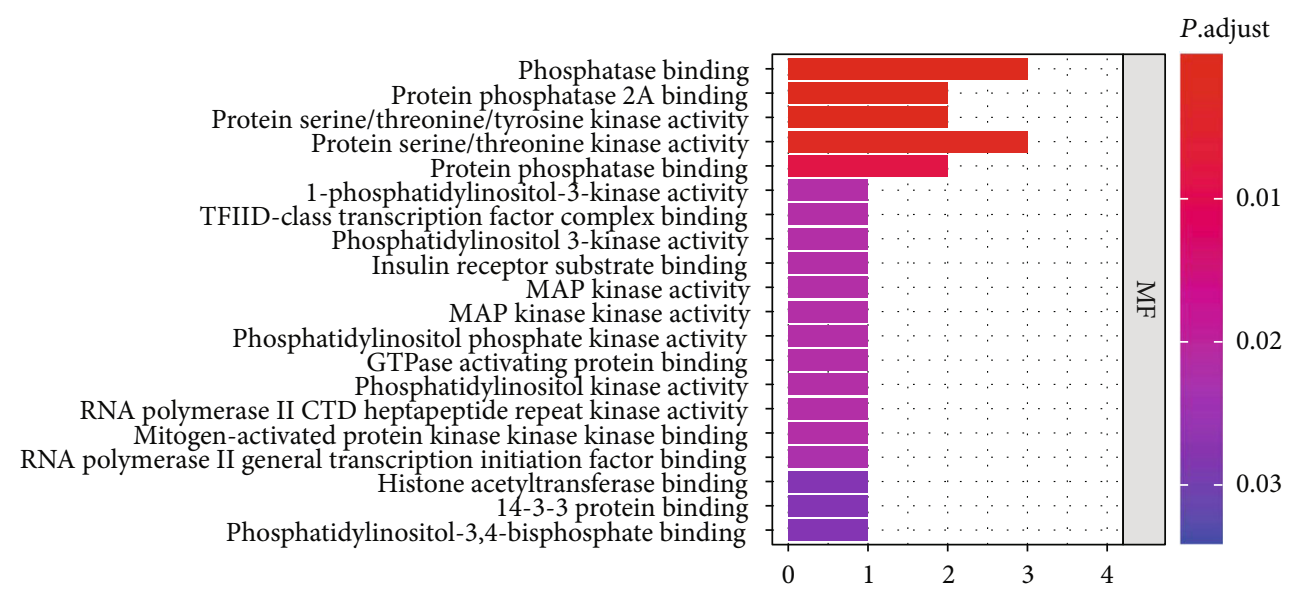

(c)

FIGURE 2: GO map of putative target genes. (a) Biological process categories. (b) Cellular component categories. (c) Molecular function categories.

MF categories were dominated by phosphatase binding and protein serine/threonine kinase activity. Various phosphatases promoted vascular remodeling and pulmonary arterial hypertension by modulating smooth muscle cell proliferation $[61,62]$. Many serine-threonine kinases, such as P-21activated kinases, PAKs, or RhoA/Rho-kinase, have been well demonstrated to promote the development of CVD $[63,64]$. It was reported that there was a strong crosstalk between oxidative stress and various CVDs, including atherosclerosis, myocardial ischemia, ischemia reperfusion injury, and drug-induced cardiotoxicity $[65,66]$. In addition, oxidative stress regulates multiple cardiovascular functions, such as cell proliferation and death [67]. KEGG pathway enrichment analysis showed TwHF may exert protective effects on CVD mainly by cancer pathways. Cancer and cardiovascular disease (CVD) share overlapping pathophysiology and risk factors as well as biological mechanisms [68]. Protein-protein interaction analysis has varied roles in driving and 


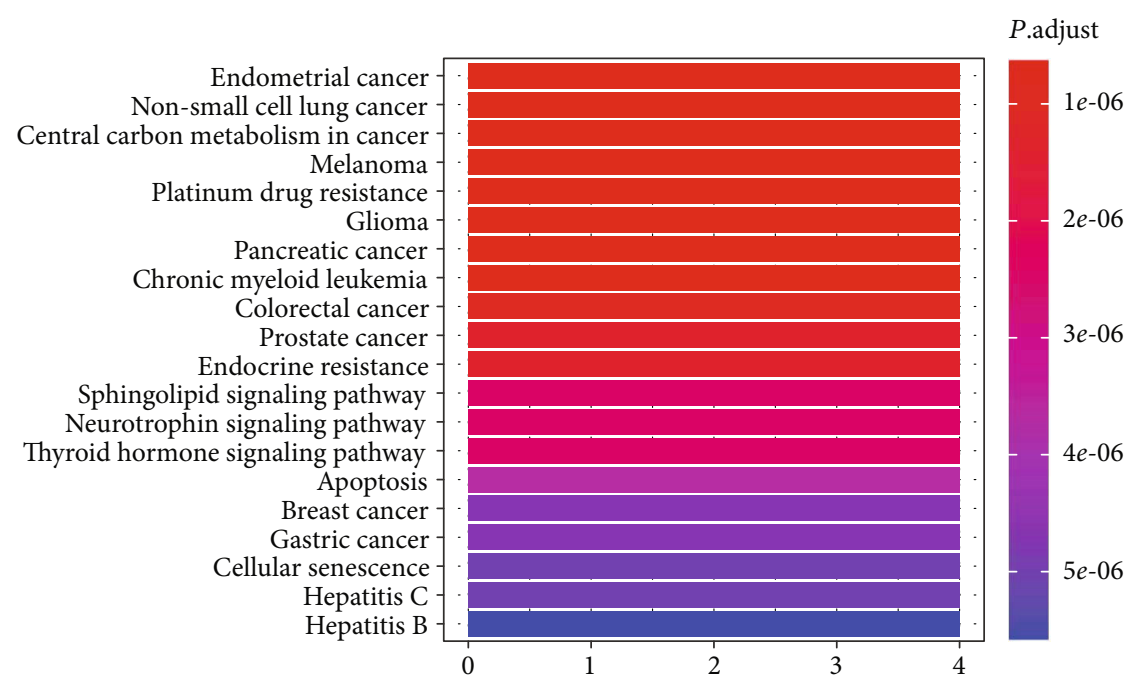

FIGURE 3: KEGG pathway analysis of putative target genes.

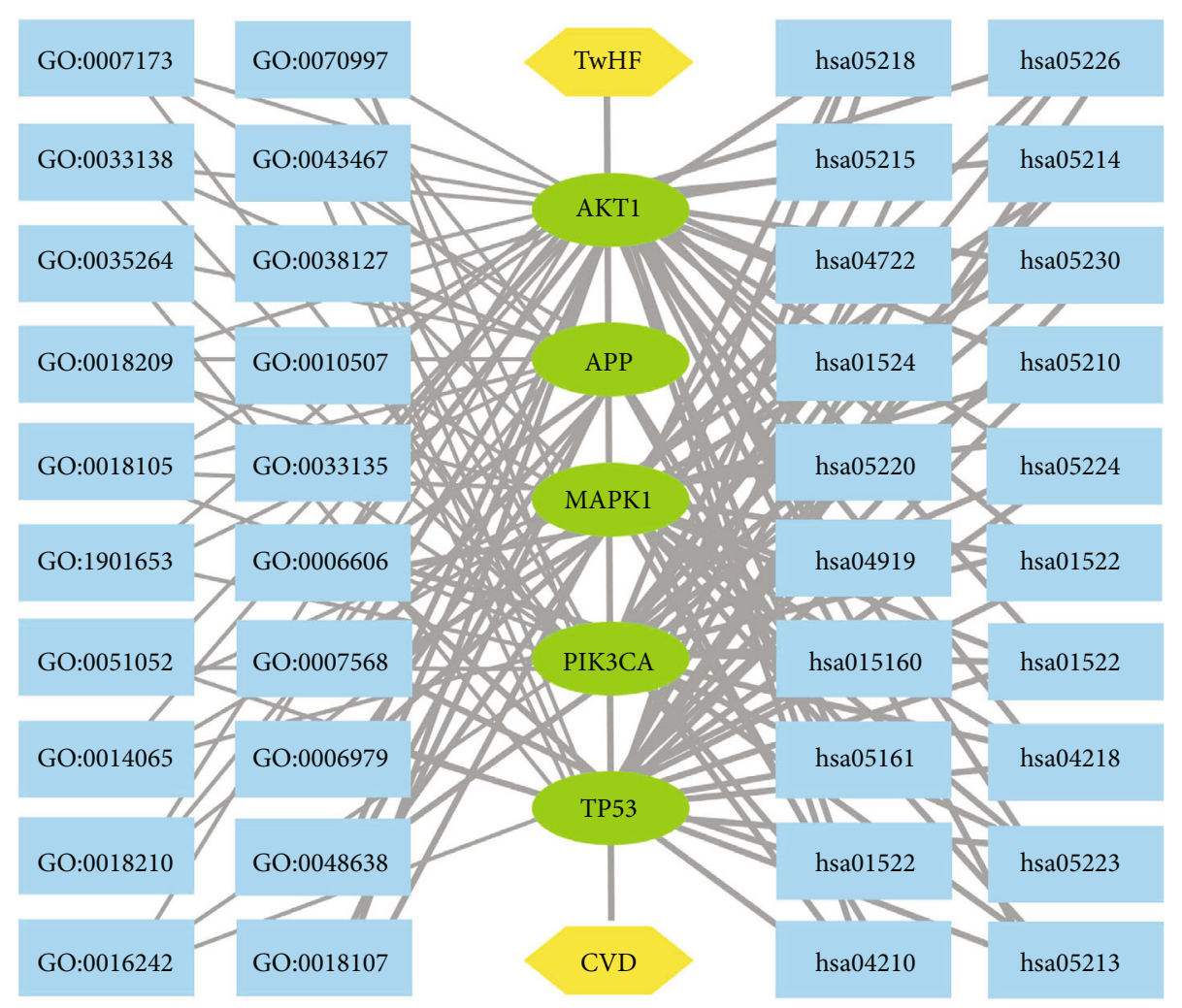

FIGURE 4: TwHF-targets-CVD-GO-KEGG network.

maintaining the growth of cancer and CVD $[69,70]$. TwHF was also determined as an efficacy treatment of multiple cancers [71-73]. In summary, GO and KEGG pathway enrichments indicated that TwHF exerting protective effects on CVD probably through modulation of the proliferation of cardiovascular system cells.

According to the screening criteria of high OB, celaxanthin, hypodiolide A, triptofordin B2, and celallocinnine were chosen for the compound-ligand interaction analysis by molecular docking to validate the effects of TwHF acting on CVD. The results of molecular docking reflected that these active compounds possess suitable anti-CVD activity. However, to verify the active properties of TwHF and the molecular target genes of anti-CVD, further experimental studies need to be performed.

\section{Conclusion}

In summary, the network pharmacology method was performed to unveil the chemical basis and investigate the action 


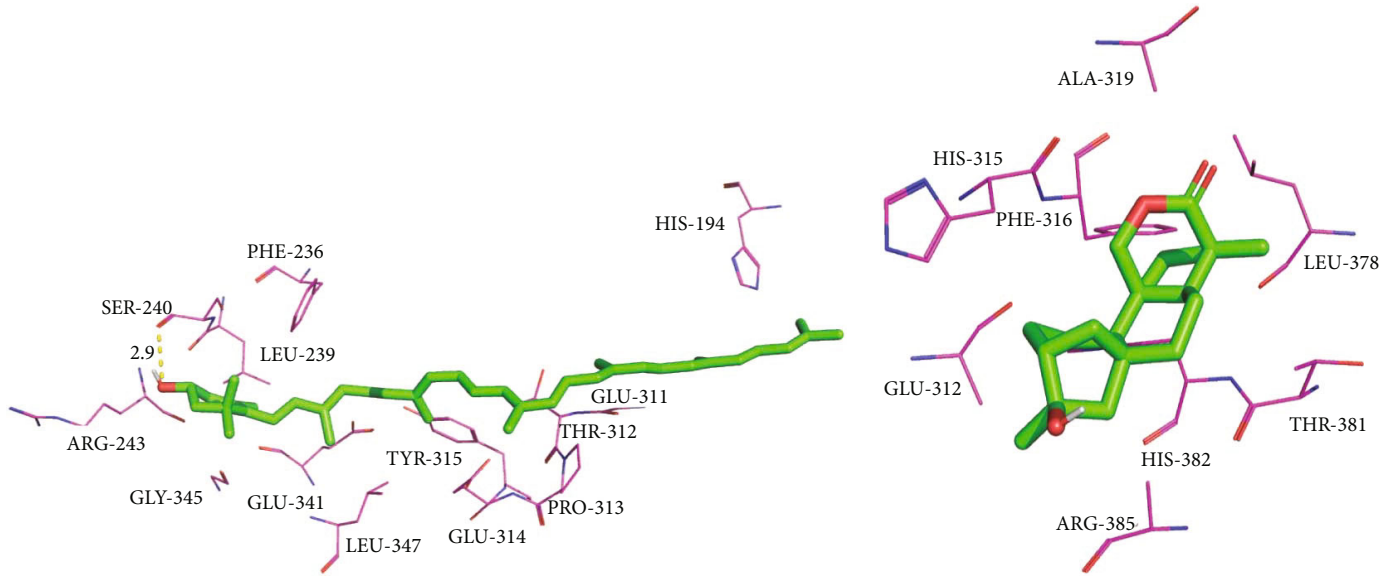

(a)

(b)

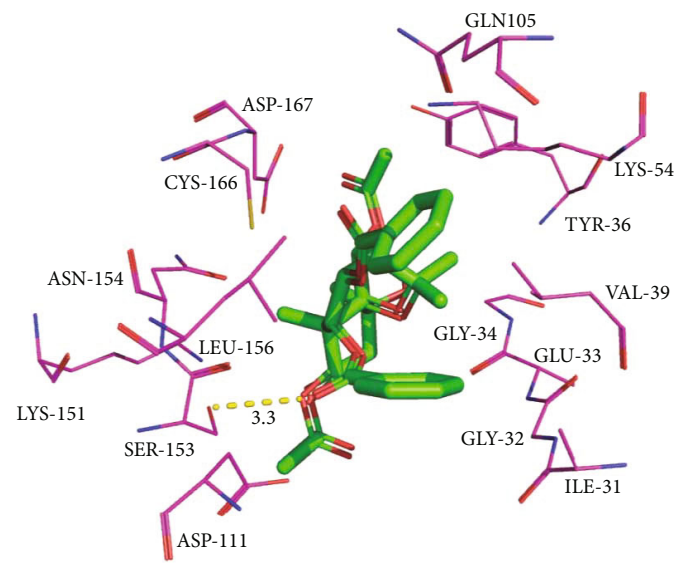

(c)

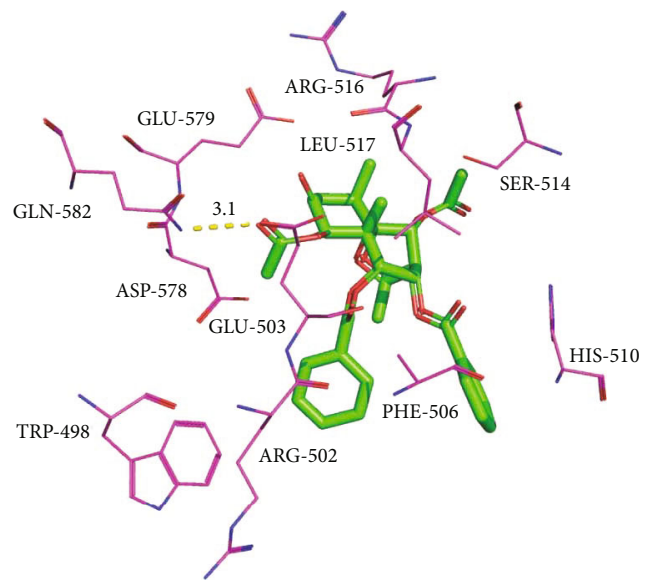

(d)

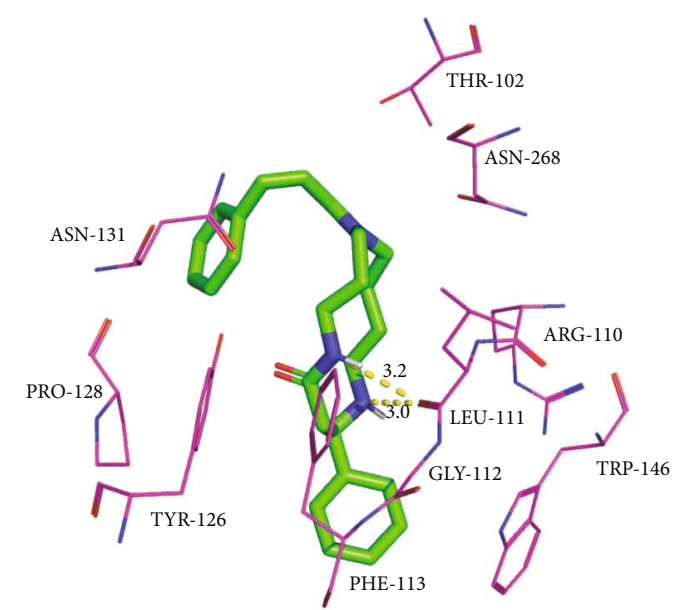

(e)

FIGURE 5: Molecular models of the binding of TwHF to the predicted targets (a) AKT1, (b) APP, (c) MAPK1, (d) PIK3CA, and (e) TP53 shown as 3D diagrams.

mechanism of TwHF on CVD. Firstly, 41 active compounds of TwHF and 5 core target genes (AKT1, APP, MAPK, PIK3CA, and TP53) of TwHF against CVD were identified. Then, based on the analysis of GO and KEGG, the cancer pathway was found to be closely associated with the protective effect of TwHF on CVD. It provides a theoretical basis and a clue for the pharmacological mechanism study of TwHF on CVD in this study.

5.1. Limitation. There are some limitations to this study. Because of the collection of targets from databases, the predicted targets might be inaccurate and specific. Other values 
of compounds might be also inaccurate, such as the OB value of triptofordin B2, which is greater than $100 \%$. These values also were predicted by chemometric method. Therefore, further experimental studies of these prediction results are needed to validate the potential applications.

\section{Data Availability}

The data used to support the findings of this study are included within the article and the supplementary files.

\section{Disclosure}

This manuscript was submitted as a preprint in the link "https://www.researchsquare.com/article/rs-151905/v1."

\section{Conflicts of Interest}

The authors report no relationships that could be construed as a conflict of interest.

\section{Authors' Contributions}

Ning Zhu designed the study and drafted and revised the manuscript. Bingwu Huang performed the research and wrote the manuscript. Chengbin Huang, Liuyan Zhu, Lina Xie, and Yi Wang collected and analyzed the data. All authors read and approved the final manuscript.

\section{Acknowledgments}

This study was supported by Zhejiang Provincial Natural Science Foundation of China under Grant no. LQ20H020011.

\section{Supplementary Materials}

Known targets of TwHF and known therapeutic targets acting on CVD were shown in Supplementary Table 1. Known protein interactions of TwHF against CVD (combined score $\geq 0.9$ ) were shown in Supplementary Table 2. (Supplementary Materials)

\section{References}

[1] E. Olvera Lopez, B. D. Ballard, and A. Jan, "Cardiovascular Disease," in StatPearls, Treasure Island (FL): StatPearls Publishing Copyright (c) 2020, StatPearls Publishing LLC, 2020.

[2] S. B. Dunbar, O. A. Khavjou, T. Bakas et al., "Projected costs of informal caregiving for cardiovascular disease: 2015 to 2035: a policy statement from the American Heart Association," Circulation, vol. 137, no. 19, pp. e558-e577, 2018.

[3] S. Bansilal, J. M. Castellano, and V. Fuster, "Global burden of CVD: focus on secondary prevention of cardiovascular disease," International journal of cardiology, vol. 201, pp. S1-S7, 2015.

[4] P. Joseph, D. Leong, M. McKee et al., "Reducing the global burden of cardiovascular disease, part 1: the epidemiology and risk factors," Circulation research, vol. 121, no. 6, pp. 677694, 2017.

[5] D. P. Leong, P. G. Joseph, M. McKee et al., "Reducing the global burden of cardiovascular disease, part 2: prevention and treatment of cardiovascular disease," Circulation research, vol. 121, no. 6, pp. 695-710, 2017.

[6] P. Hao, F. Jiang, J. Cheng, L. Ma, Y. Zhang, and Y. Zhao, "Traditional Chinese medicine for cardiovascular disease: evidence and potential mechanisms," Journal of the American College of Cardiology, vol. 69, no. 24, pp. 2952-2966, 2017.

[7] X. Zhao, Z. Liu, Z. Ren et al., “Triptolide inhibits pancreatic cancer cell proliferation and migration via down-regulating PLAU based on network pharmacology of _Tripterygium wilfordii Hook F_," European journal of pharmacology, vol. 880, p. $173225,2020$.

[8] M. Xue, Z. Z. Jiang, T. Wu et al., "Anti-inflammatory effects and hepatotoxicity of Tripterygium-loaded solid lipid nanoparticles on adjuvant-induced arthritis in rats," Phytomedicine, vol. 19, no. 11, pp. 998-1006, 2012.

[9] B. S. Choi, K. Sapkota, S. Kim, H. J. Lee, H. S. Choi, and S. J. Kim, "Antioxidant activity and protective effects of Tripterygium regelii extract on hydrogen peroxide-induced injury in human dopaminergic cells, SH-SY5Y," Neurochemical Research, vol. 35, no. 8, pp. 1269-1280, 2010.

[10] W. H. Peng, G. L. Chen, Y. Zhou, S. Y. Fan, D. L. Fu, and Y. Wang, "Study of expression of circulating inflammatory factors in ACS rats with low dose of Tripterygium Wilfordii," Hellenic Journal of Cardiology, vol. 59, no. 1, pp. 4647, 2018.

[11] X. Li, N. Wu, L. Zou, and D. Jia, "Protective effect of celastrol on myocardial ischemia-reperfusion injury," Anatolian journal of cardiology, vol. 18, no. 6, pp. 384-390, 2017.

[12] R. Li, K. Lu, Y. Wang et al., "Triptolide attenuates pressure overload-induced myocardial remodeling in mice via the inhibition of NLRP3 inflammasome expression," Biochemical and biophysical research communications, vol. 485, no. 1, pp. 6975, 2017.

[13] H. Yu, L. Shi, S. Zhao et al., "Triptolide attenuates myocardial ischemia/reperfusion injuries in rats by inducing the activation of Nrf2/HO-1 defense pathway," Cardiovascular toxicology, vol. 16, no. 4, pp. 325-335, 2016.

[14] C. Lu, X. Yu, K. Zuo et al., "Tripterine treatment improves endothelial progenitor cell function via integrin-linked kinase," Cellular physiology and biochemistry, vol. 37, no. 3, pp. 1089-1103, 2015.

[15] B. Han, C.-Q. Ge, H.-G. Zhang et al., "Effects of tripterygium glycosides on restenosis following endovascular treatment," Molecular medicine reports, vol. 13, no. 6, pp. 4959-4968, 2016.

[16] A. L. Hopkins, "Network pharmacology," Nature biotechnology, vol. 25, no. 10, pp. 1110-1111, 2007.

[17] C. Lu, Z. Bing, Z. Bi et al., "Top-100 most cited publications concerning network pharmacology: a bibliometric analysis," Evidence-based complementary and alternative medicine, vol. 2019, Article ID 1704816, 7 pages, 2019.

[18] P. Li, J. Chen, J. Wang et al., "Systems pharmacology strategies for drug discovery and combination with applications to cardiovascular diseases," Journal of Ethnopharmacology, vol. 151, no. 1, pp. 93-107, 2014.

[19] J. Ru, P. Li, J. Wang et al., "TCMSP: a database of systems pharmacology for drug discovery from herbal medicines," Journal of cheminformatics, vol. 6, no. 1, 2014.

[20] F. Liu, Y. Li, M. Li et al., "Study on mechanism of iridoid glycosides derivatives from Fructus Gardeniae in Jiangxi Province by network pharmacology," Evidence-based complementary 
and alternative medicine, vol. 2020, Article ID 4062813, 12 pages, 2020.

[21] L. Huang, D. Xie, Y. Yu et al., "TCMID 2.0: a comprehensive resource for TCM," Nucleic acids research, vol. 46, no. D1, pp. D1117-d1120, 2018.

[22] J. Xu, F. Wang, J. Guo et al., "Pharmacological mechanisms underlying the neuroprotective effects of Alpinia oxyphylla Miq. on Alzheimer's disease," International journal of molecular sciences, vol. 21, no. 6, p. 2071, 2020.

[23] A. Daina, O. Michielin, and V. Zoete, "SwissTargetPrediction: updated data and new features for efficient prediction of protein targets of small molecules," Nucleic acids research, vol. 47, no. W1, pp. W357-w364, 2019.

[24] D. S. Wishart, Y. D. Feunang, A. C. Guo et al., "DrugBank 5.0: a major update to the DrugBank database for 2018," Nucleic acids research, vol. 46, no. D1, pp. D1074-d1082, 2018.

[25] J. S. Amberger and A. Hamosh, "Searching Online Mendelian Inheritance in Man (OMIM): a knowledgebase of human genes and genetic phenotypes," Current protocols in bioinformatics, vol. 58, no. 1, 2017.

[26] G. Stelzer, N. Rosen, I. Plaschkes et al., "The GeneCards suite: from gene data mining to disease genome sequence analyses," Current protocols in bioinformatics, vol. 54, no. 1, 2016.

[27] M. Kuhn, D. Szklarczyk, A. Franceschini et al., "STITCH 2: an interaction network database for small molecules and proteins," Nucleic acids research, vol. 38, suppl_1, pp. D552D556, 2010.

[28] J. A. C. K. Blake, M. E. Dolan, H. J. Drabkin et al., "Gene ontology consortium: going forward," Nucleic acids research, vol. 43, pp. D1049-D1056, 2015.

[29] M. Kanehisa, M. Furumichi, M. Tanabe, Y. Sato, and K. Morishima, "KEGG: new perspectives on genomes, pathways, diseases and drugs," Nucleic acids research, vol. 45, no. D1, pp. D353-d361, 2017.

[30] P. Shannon, A. Markiel, O. Ozier et al., "Cytoscape: a software environment for integrated models of biomolecular interaction networks," Genome Research, vol. 13, no. 11, pp. 24982504, 2003.

[31] R. Li, C. Guo, Y. Li, Z. Qin, and W. Huang, "Therapeutic targets and signaling mechanisms of vitamin $\mathrm{C}$ activity against sepsis: a bioinformatics study," Briefings in bioinformatics, vol. 22, 2021.

[32] J. Zhang, H. Li, Y. Zhang, C. Zhao, Y. Zhu, and M. Han, "Uncovering the pharmacological mechanism of stemazole in the treatment of neurodegenerative diseases based on a network pharmacology approach," International journal of molecular sciences, vol. 21, no. 2, p. 427, 2020.

[33] X. Song, Y. Zhang, E. Dai, L. Wang, and H. Du, "Prediction of triptolide targets in rheumatoid arthritis using network pharmacology and molecular docking," International immunopharmacology, vol. 80, 2020.

[34] W. Zhou, Y. Wang, A. Lu, and G. Zhang, "Systems pharmacology in small molecular drug discovery," International journal of molecular sciences, vol. 17, no. 2, p. 246, 2016.

[35] S. Ye, W. Luo, Z. A. Khan et al., "Celastrol attenuates angiotensin II-induced cardiac remodeling by targeting STAT3," Circulation research, vol. 126, no. 8, pp. 1007-1023, 2020.

[36] S. der Sarkissian, J. F. Cailhier, M. Borie et al., "Celastrol protects ischaemic myocardium through a heat shock response with up-regulation of haeme oxygenase-1," British journal of pharmacology, vol. 171, no. 23, pp. 5265-5279, 2014.
[37] M. Cheng, G. Wu, Y. Song et al., "Celastrol-induced suppression of the MiR-21/ERK signalling pathway attenuates cardiac fibrosis and dysfunction," Cellular physiology and biochemistry, vol. 38, no. 5, pp. 1928-1938, 2016.

[38] F. Liu, H. Zhang, Y. Li, and X. Lu, "Nobiletin suppresses oxidative stress and apoptosis in H9c2 cardiomyocytes following hypoxia/reoxygenation injury," European journal of pharmacology, vol. 854, pp. 48-53, 2019.

[39] B. F. Zhang, H. Jiang, J. Chen et al., "Nobiletin ameliorates myocardial ischemia and reperfusion injury by attenuating endoplasmic reticulum stress-associated apoptosis through regulation of the PI3K/AKT signal pathway," International immunopharmacology, vol. 73, pp. 98-107, 2019.

[40] X. Guo, M. Xue, C. J. Li et al., "Protective effects of triptolide on TLR4 mediated autoimmune and inflammatory response induced myocardial fibrosis in diabetic cardiomyopathy," Journal of ethnopharmacology, vol. 193, pp. 333-344, 2016.

[41] Z. Liang, S. Leo, H. Wen, M. Ouyang, W. Jiang, and K. Yang, "Triptolide improves systolic function and myocardial energy metabolism of diabetic cardiomyopathy in streptozotocininduced diabetic rats," BMC cardiovascular disorders, vol. 15, no. 1, 2015.

[42] R. Negrão, D. Duarte, R. Costa, and R. Soares, "Isoxanthohumol modulates angiogenesis and inflammation via vascular endothelial growth factor receptor, tumor necrosis factor alpha and nuclear factor kappa B pathways," BioFactors, vol. 39, no. 6, pp. 608-622, 2013.

[43] C. Li, Y. Liu, Z. Xie, Q. Lu, and S. Luo, "Stigmasterol protects against Ang II-induced proliferation of the A7r5 aortic smooth muscle cell-line," Food \& function, vol. 6, no. 7, pp. 2266-2272, 2015.

[44] H. Feng, J. Cao, G. Zhang, and Y. Wang, "Kaempferol attenuates cardiac hypertrophy via regulation of ASK1/MAPK signaling pathway and oxidative stress," Planta Medica, vol. 83, no. 10, pp. 837-845, 2017.

[45] L. Zhang, Z. Guo, Y. Wang, J. Geng, and S. Han, “The protective effect of kaempferol on heart via the regulation of $\mathrm{Nrf} 2$, NF- $\kappa \beta$, and PI3K/Akt/GSK-3 $\beta$ signaling pathways in isoproterenol-induced heart failure in diabetic rats," Drug development research, vol. 80, no. 3, pp. 294-309, 2019.

[46] P. Abeyrathna and Y. Su, "The critical role of Akt in cardiovascular function," Vascular pharmacology, vol. 74, pp. 38-48, 2015.

[47] G. Yu, Z. Luo, Y. Zhou et al., "Uncovering the pharmacological mechanism of Carthamus tinctorius L. on cardiovascular disease by a systems pharmacology approach," Biomedicine \& pharmacotherapy, vol. 117, 2019.

[48] S. J. Leevers, B. Vanhaesebroeck, and M. D. Waterfield, "Signalling through phosphoinositide 3-kinases: the lipids take centre stage," Current opinion in cell biology, vol. 11, no. 2, pp. 219-225, 1999.

[49] C. L. Carpenter, B. C. Duckworth, K. R. Auger, B. Cohen, B. S. Schaffhausen, and L. C. Cantley, "Purification and characterization of phosphoinositide 3-kinase from rat liver.," The Journal of biological chemistry, vol. 265, no. 32, pp. 19704-19711, 1990.

[50] B. A. McLean, V. B. Patel, P. Zhabyeyev et al., "PI3K $\alpha$ pathway inhibition with doxorubicin treatment results in distinct biventricular atrophy and remodeling with right ventricular dysfunction," Journal of the American Heart Association, vol. 8, no. 9, p. e010961, 2019. 
[51] The Cancer Genome Atlas Network, "Comprehensive molecular portraits of human breast tumours," Nature, vol. 490, no. 7418, pp. 61-70, 2012.

[52] G. Pearson, F. Robinson, T. Beers Gibson et al., "Mitogen-activated protein (MAP) kinase pathways: regulation and physiological functions," Endocrine reviews, vol. 22, no. 2, pp. 153183, 2001.

[53] Y. Wang, X. Zhang, L. Gao et al., "Cortistatin exerts antiproliferation and antimigration effects in vascular smooth muscle cells stimulated by Ang II through suppressing ERK1/2, p38 MAPK, JNK and ERK5 signaling pathways," Annals of translational medicine, vol. 7, no. 20, p. 561, 2019.

[54] Y. Taniyama, M. Ushio-Fukai, H. Hitomi et al., "Role of p38 MAPK and MAPKAPK-2 in angiotensin II-induced Akt activation in vascular smooth muscle cells," American journal of physiology Cell physiology, vol. 287, no. 2, pp. C494-C499, 2004.

[55] Y. Kyotani, J. Zhao, S. Tomita et al., "Olmesartan inhibits angiotensin II-induced migration of vascular smooth muscle cells through Src and mitogen-activated protein kinase pathways," Journal of Pharmacological Sciences, vol. 113, no. 2, pp. 161-168, 2010.

[56] C. Visconte, J. Canino, G. F. Guidetti et al., “Amyloid precursor protein is required for in vitro platelet adhesion to amyloid peptides and potentiation of thrombus formation," Cellular signalling, vol. 52, pp. 95-102, 2018.

[57] I. Canobbio, C. Visconte, S. Momi et al., "Platelet amyloid precursor protein is a modulator of venous thromboembolism in mice," Blood, vol. 130, no. 4, pp. 527-536, 2017.

[58] V. Kolovou, A. Tsipis, C. Mihas et al., "Tumor protein p53 (TP53) gene and left main coronary artery disease," Angiology, vol. 69 , no. 8, pp. 730-735, 2018.

[59] K. Lemmens, K. Doggen, and G. W. De Keulenaer, "Role of neuregulin-1/ErbB signaling in cardiovascular physiology and disease: implications for therapy of heart failure," Circulation, vol. 116, no. 8, pp. 954-960, 2007.

[60] O. Odiete, M. F. Hill, and D. B. Sawyer, "Neuregulin in cardiovascular development and disease," Circulation research, vol. 111, no. 10, pp. 1376-1385, 2012.

[61] J. Huang, C. Cai, T. Zheng et al., "Endothelial scaffolding protein ENH (enigma homolog protein) promotes PHLPP2 (pleckstrin homology domain and leucine-rich repeat protein phosphatase 2)-mediated dephosphorylation of AKT1 and eNOS (endothelial NO synthase) promoting vascular remodeling," Arteriosclerosis, thrombosis, and vascular biology, vol. 40, no. 7, pp. 1705-1721, 2020.

[62] L. Kovacs, Y. Cao, W. Han et al., "PFKFB3 in smooth muscle promotes vascular remodeling in pulmonary arterial hypertension," American journal of respiratory and critical care medicine, vol. 200, no. 5, pp. 617-627, 2019.

[63] D. M. Taglieri, M. Ushio-Fukai, and M. M. Monasky, "P21activated kinase in inflammatory and cardiovascular disease," Cellular signalling, vol. 26, no. 9, pp. 2060-2069, 2014.

[64] H. Shimokawa, S. Sunamura, and K. Satoh, "RhoA/Rho-kinase in the cardiovascular system," Circulation research, vol. 118, no. 2, pp. 352-366, 2016.

[65] X. Su, C. Yuan, L. Wang et al., "The beneficial effects of saffron extract on potential oxidative stress in cardiovascular diseases," Oxidative medicine and cellular longevity, vol. 2021, Article ID 6699821, 14 pages, 2021.
[66] T. Donia and A. Khamis, "Management of oxidative stress and inflammation in cardiovascular diseases: mechanisms and challenges," Environmental science and pollution research international, vol. 28, no. 26, pp. 34121-34153, 2021.

[67] E. Dubois-Deruy, V. Peugnet, A. Turkieh, and F. Pinet, "Oxidative stress in cardiovascular diseases," Antioxidants, vol. 9, no. 9 , p. $864,2020$.

[68] S. Tiwari and U. N. Dwivedi, "Discovering innovative drugs targeting both cancer and cardiovascular disease by shared protein-protein interaction network analyses," Omics: a journal of integrative biology, vol. 23, no. 9, pp. 417-425, 2019.

[69] A. A. Ivanov, F. R. Khuri, and H. Fu, "Targeting proteinprotein interactions as an anticancer strategy," Trends in pharmacological sciences, vol. 34, no. 7, pp. 393-400, 2013.

[70] A. Sarajlić, V. Janjić, N. Stojković, D. Radak, and N. Pržulj, "Network topology reveals key cardiovascular disease genes," PLoS One, vol. 8, no. 8, article e71537, 2013.

[71] C. Zhang, X. J. He, L. Li, C. Lu, and A. P. Lu, "Effect of the natural product triptolide on pancreatic cancer: a systematic review of preclinical studies," Frontiers in pharmacology, vol. $8,2017$.

[72] L. Wen, Y. Chen, L. L. Zeng et al., "Triptolide induces cell apoptosis by targeting $\mathrm{H} 3 \mathrm{~K} 4 \mathrm{me} 3$ and downstream effector proteins in KM3 multiple myeloma cells," Current pharmaceutical biotechnology, vol. 17, no. 2, pp. 147-160, 2015.

[73] O. Fuchs, "Transcription factor NF- $\kappa$ B inhibitors as single therapeutic agents or in combination with classical chemotherapeutic agents for the treatment of hematologic malignancies," Current molecular pharmacology, vol. 3, no. 3, pp. 98$122,2010$. 